\title{
EDITORIAL
}

\section{The herbal ephedra and caffeine debate continues}

DOI: $10.1038 / \mathrm{sj} / \mathrm{ijo} / 0802043$

The issue of the use of herbal supplements containing ephedra and caffeine for weight loss has become highly controversial within the obesity community. In this issue, a manuscript by Boozer et al (page 593-604) reports a randomized, six-month trial in 167 subjects on an ephedracaffeine herbal supplement $v s$ placebo. This group previously reported an eight week trial using an herbal supplement in the International Journal of Obesity (Int J Obes Relat Metab Disord 2001; 25: 316-324). The previous manuscript generated a series of letters to the editor, some of which were critical to highly critical of the authors and the Editors, but without, in my opinion, good scientific foundation. A major stimulus to these letters was the use of the original Boozer et al article by supplement manufacturers to advertise ephedra-caffeine supplements as safe and effective. The Editors invited Dr A Dulloo to write an editorial putting into perspective the original paper, the use of the original paper, the letters to the editor, and the scientific background of the use of ephedrine and caffeine for weight loss. However, with the arrival of a second manuscript by Boozer et al reporting on the longer trial, the Dulloo editorial was held until a decision could be made on this manuscript. With acceptance of this manuscript after favourable reviews, the Editors asked Dulloo to revisit the topic and to comment on both papers. This editorial is on pages 590-592.
Almost all opinion papers in the scientific and lay press lament the lack of data on dietary supplements and conclude with a call for additional research. The Editors appreciate the controversial nature of this research, but believe that it is critical to publish well done trials of the use of dietary supplements. Neither the authors nor the International Journal of Obesity condone the use of either of the Boozer et al papers on ephedra-caffeine to promote the use of herbal supplements to the public. As carefully pointed out by both Boozer and Dulloo, the subjects selected for these studies were carefully selected and were free of medical problems and other contraindications to the use of drugs that affect the heart and central nervous system. Herbal supplement manufacturers should act responsibly in advertising their supplements, and the lay public should be aware that these papers do not assure the safety, or even the efficacy, of herbal supplements containing ephedra-caffeine in individuals who differ from the carefully selected study subjects. The lay public should use ephedra-caffeine supplements with great caution and, in the opinion of this Editor, only under the supervision of a physician.

RL Atkinson, MD Editor, International Journal of Obesity 